\title{
Changing bodies changes minds: owning another body affects social cognition
}

Lara Maister $^{1}$, Mel Slater ${ }^{2,3,5}$, Maria V. Sanchez-Vives ${ }^{4,5}$, \& Manos Tsakiris ${ }^{1 *}$

${ }^{1}$ Department of Psychology, Royal Holloway University of London, Egham, Surrey, UK

${ }^{2}$ Faculty of Psychology, Universitat de Barcelona, Barcelona, Spain

${ }^{3}$ Department of Computer Science, University College London, London, UK.

${ }^{4}$ Institut d'Investigacions Biomediques August Pi i Sunyer (IDIBAPS), Barcelona, Spain

${ }^{5}$ Institució Catalana Recerca i Estudis Avançats (ICREA), Barcelona, Spain

*corresponding author: Tsakiris, M. (manos.tsakiris@rhul.ac.uk)

This paper is published as: Maister L, Slater M, Sanchez-Vives MV \& Tsakiris M (2014).

Changing bodies changes minds: owning another body affects social cognition. Trends in Cognitive Sciences. In press / doi:10.1016/j.tics.2014.11.001 


\section{Abstract}

Research on stereotypes demonstrates how existing prejudice affects the way we process

2 outgroups. Recent studies have considered whether it is possible to change our implicit social bias

3 by experimentally changing the relation between the self and outgroups. In a number of

4 experimental studies, participants have been exposed to bodily illusions that induced ownership

5 over a body different to their own with respect to gender, age or race. Ownership of an outgroup

6 body has been found to be associated with a significant reduction in implicit biases against that

7 outgroup. We propose that these changes occur via a process of self-association that first takes place

8 in the physical, bodily domain as an increase in perceived physical similarity between self and

9 outgroup member. This self-association then extends to the conceptual domain, leading to a

10 generalization of positive self-like associations to the outgroup.

11 Keywords: body ownership, racial biases, implicit attitudes, social cognition, bodily illusions, 12 immersive virtual reality

Highlights

- Multisensory correlations can induce illusory ownership of another person's body.

- Ownership can thus be induced over a body of a different race, age, or gender.

- Incorporating a body belonging to a social outgroup changes implicit social biases.

- The multisensory experience of the body underpins higher-level social attitudes. 


\section{Body representations of self and other.}

Embodied accounts of social cognition suggest that the way in which we perceive others' bodies in relation to our own plays a crucial role in sociocognitive processing [1-7]. The perception of bodily states in others can activate similar bodily states in the self, and this is taken as evidence that our representations of our own bodies and those of others can partially overlap. These shared body representations are thought to form the fundamental basis of empathy and our understanding of others' emotions and actions. Interestingly, the activation of shared body representations is modulated by whether the person being observed is an ingroup or an outgroup member. For example, when we observe an individual of a different race to ourselves experiencing a specific bodily state, such as touch or pain, we show a reduced sharing of that bodily state. Furthermore, this reduction is modulated by our implicit social attitudes towards that racial group; the more negatively biased we are against members of that race, the less overlap between our representations of their bodies and our own [see below; 3-4,8].

Until recently, research in this area has focused on how existing social bias and prejudice affect the way we process outgroup members [3-6], rather than investigating the potential malleability of our ingroup/outgroup classifications. A series of recent studies have successfully filled this gap [9-14] by asking whether and how it is possible to change implicit social attitudes towards outgroups [15] by experimentally increasing the sharing of body representations [16]. Taken together, the findings show that changes in the mental representation of one's own body affect the perceived similarity between one's own body and that of an outgroup, resulting in significant changes in implicit biases. We here present a possible mechanism underlying these changes, which has far-reaching implications for our understanding of the development and malleability of social attitudes, and the crucial role of basic body representations in these processes.

\section{Racial Biases in Brain, Behaviour and the Body}


A rapidly growing literature suggests that the body is central to our understanding of others. Neurocognitive studies into the 'mirror neuron system' have shown that we activate similar brain regions both when we observe a bodily state in others and when we experience that bodily state ourselves [17], reflecting an overlap between self and other bodily representations in the brain [18]. Evidence now suggests that this bodily resonance (see Glossary) can afford us a unique, first-person understanding of the experiences of others and is central to a number of social processes [7] including intention understanding [19], empathy [20], and emotion recognition [21]. Importantly, recent studies have revealed that social group categorisation, such as that based on racial group membership, can have a strong impact on the extent to which we resonate with others' bodily states.

Racial group membership is a salient distinguishing factor between individuals, and has long been known to strongly impact human social behaviours and attitudes. For example, we tend to show implicit biases towards members of our own race and against those of other races, even when we don’t hold any explicitly biased attitudes. These implicit racial biases can be measured behaviourally using an implicit association task (IAT: See Glossary [15]), but also can be seen at the neural level in the form of distinct patterns of brain activity [2]. Intriguingly, bodily resonance is modulated by whether the other person being observed is a member of a racial ingroup or outgroup [3-8]. For example, viewing a face being touched enhances the perception of touch on one’s own face, but this effect, known as the Visual Remapping of Touch, is not present when the observed face belongs to a racial outgroup member [5]. In the motor domain, participants show reduced vicarious activation of the motor cortex when observing actions performed by a racial outgroup member as compared to an ingroup member $[4,8]$, and show decreased neural and motor responses when viewing racial outgroup members in pain $[3,6]$. Furthermore, this diminished neural resonance with the racial outgroup has been found to directly correlate with participants' negative implicit racial biases [3]. Until recently, research in this area has focussed on how bodily resonance is affected by existing racial attitudes. Could this relationship, in fact, be bidirectional? In other words, could 
existing racial attitudes be modulated by the experimental manipulation of shared body

representations? A series of recent studies has employed a range of multisensory methods to manipulate body ownership and has revealed striking effects on implicit racial attitudes.

\section{From body ownership to social cognition: Constraints and consequences}

Over the last twenty years, advances in experimental psychology, cognitive neuroscience and virtual reality have allowed scientists to experiment with a fundamental element of selfawareness, the sense of body ownership (see Glossary), using a range of bodily illusions, such as the Rubber Hand Illusion [22], the Full Body Illusion [23-25] and the Enfacement Illusion [26] (see Box 1 for descriptions). These successful manipulations aptly demonstrated the malleability of the mental representation of one's body and identity.

$$
\text { Having established the behavioural and neural correlates of these multisensory-induced }
$$
changes in body ownership, attention has turned towards the potential social constraints, as well as the social consequences, of such changes. Importantly, illusions of body ownership were revealed to be surprisingly impervious to social and perceptual distinctions. Several studies, using a variety of methods, successfully induced a sense of body ownership over bodies of different race- $[9,10,12-$ 14,27, 28, 29], age- [11], size- [11,30,31,32] and gender-groups [25]. Furthermore, in the case where the different body depicted an outgroup person, the acquired ownership did not depend on pre-existing levels of implicit outgroup bias; participants experienced ownership over another's body regardless of their levels of negative implicit attitudes towards the other's social group [13]. This provides an interesting contrast with the findings already discussed, which show that shared body representations, in the absence of experimental manipulations that prime the self-relevance of the observed body, are indeed greatly influenced by factors such as racial attitudes. However, the manipulations used to induce bodily illusions involve highly salient multisensory cues which are strongly predictive of body ownership, and thus may override top-down modulations by social attitudes [9-13]. Conversely, in the absence of these powerful multisensory cues, the effects of social attitudes on bodily resonance with others may emerge. 
Despite being relatively impervious to social factors, the experimental modulation of body ownership was found to have a number of intriguing effects on social cognition. After synchronous multisensory stimulation on the face (see Enfacement Illusion, Box 1), participants rated the other's face as more attractive, more physically similar to their own, and they were also more likely to conform to the other's opinions [33]. Effects were also seen in the emotional domain; the enfacement illusion improved recognition of the other's emotions, with a specific increase in sensitivity to fearful facial expressions [34].

\section{Changing your body changes your mind}

Although changes in body ownership were found to affect social processing of 'embodied' individuals, the question of whether these changes could affect implicit biases against outgroups remained unanswered. In the first study to test this [9], participants' implicit racial attitudes were measured before and after they experienced a rubber hand illusion with a hand of a different racial group (see Fig.1). To begin, light-skinned Caucasian participants completed a skin-color IAT to assess their implicit attitudes towards individuals with dark skin. In a separate session, synchronous multisensory stimulation was used to induce the feeling that a dark-skinned hand belonged to them, before their implicit attitudes were measured for a second time. As shown previously [13], participants experienced the other-race hand as their own and body ownership occurred regardless of their implicit attitudes towards that race. Importantly, participants showed a significant decrease in negative implicit attitudes towards dark skin, which correlated with the strength of ownership experienced over the other-race hand. The more intense the participants' illusion of ownership over the dark-skinned rubber hand, the more positive their implicit racial attitudes became.

In a similar way, using an immersive virtual reality set-up [10], embodiment of lightskinned people in a dark-skinned virtual body reduced their implicit racial bias as measured by a racial IAT. To control for the effects of mere perceptual difference between the body of the avatar and participants' actual bodies, in another condition participants embodied a purple-skinned body, but this condition did not produce any changes in racial bias (see Fig.2) even though the subjective 
121 illusion of body ownership was strong and not significantly different from embodiment of the light122 or dark-skinned bodies.

Importantly, such changes in body ownership to incorporate an outgroup body also increase 'bodily resonance’ with that outgroup. As previously discussed, our perceptual and neural resonance with others’ bodily experiences is significantly reduced when observing an outgroup member [3-6,8]. An example of this can be seen in the Visual Remapping of Touch effect, a phenomenon whereby our tactile sensitivity is enhanced when observing another person being touched. This effect, thought to be evidence of somatosensory resonance with others, is significantly reduced when the observed individual is a member of a racial or political outgroup [5]. In a recent study, an Enfacement Illusion was rapidly induced by exposing participants to two minutes of multisensory stimulation whilst viewing an out-group member's face [14]. Immediately afterwards, participants’ tactile sensitivity was measured whilst they observed the out-group member's face being touched. Results showed that the experience of body ownership over the outgroup member's face had increased the Visual Remapping of Touch effect up to the level normally associated with a same-race individual.

A further study investigated implicit attitudes towards age [11] using an immersive virtual reality setup similar to that employed in previous studies [10]. Embodying an avatar representing a 138 4-year-old child resulted in a bias towards associating the self with child-like compared to adult-like 139 categorizations, as measured using an IAT. This study was notable because it demonstrated a role of 140 the self-association in attitude change, whereas previous research [9,10] had investigated more 141 generic positive or negative associations with the embodied social group. This can provide us with 142 the beginnings of a mechanism to explain how exactly 'changing your body' is able to also 'change 143 your mind'. 
How can a change in the perception of a purely bodily aspect of the self ultimately alter not only associations with a higher-level concept of the self [11], but also generalize to the affective and social processing of others? We argue that these changes occur via a process of self-association, first in the physical, bodily domain as an increase in perceived physical similarity between self and outgroup member, and then in the conceptual domain, leading to a generalization of positive selflike associations to the outgroup.

The first relevant finding to support our argument is that experimentally induced modulations of body ownership enhance perceived physical similarity between self and other. For example, after the rubber hand illusion, participants rated the rubber hand as more physically similar to their own [26]. In a more objective quantification of a comparable effect, participants accepted morphed photos of faces with a higher percentage of the other as depicting themselves after experiencing an enfacement illusion, suggesting that the participants' stored representations of their own faces were altered to incorporate aspects of the other person [26,35,36].

We suggest that this increased perceptual similarity between oneself and an outgroup member leads to a new association being formed between the self-concept and that outgroup. For this to occur, two processes are necessary. First, the perceptual self-similarity of the outgroup must activate the self-concept. We know that even subliminal exposure to images of one's own body automatically activates positive self-associations $[37,38]$ and thus we argue that perceptions of selfsimilar bodies may activate self-associations in the same way. The second required step is for the positive evaluations associated with the self-concept to be generalized to the outgroup, by virtue of their perceptual similarity to the self. In support of this, the classical conditioning literature has long posited that associative learning of likes and dislikes are based on perceptual similarity, and that this can occur outside of awareness [39,40]. This process of evaluative conditioning has been shown to extend to social stimuli; individuals rapidly and unintentionally generalize affective processing to individuals who look physically similar [41,42]. 
We propose that, because of a newly established physical similarity between self and outgroup, the conceptual representations of self and outgroup also become linked. Via a process of evaluative generalization, the positive evaluations associated with the self-concept now extend to the embodied outgroup. This results in the outgroup not only being associated with the self-concept, as already shown by [11], but also with positive concepts more generally, as shown by $[9,10]$. This mechanism can be thought of as maintaining consistency between the multifaceted aspects of self (personality, attitudes and behaviors) and the body representation following the updates of the sense of body ownership (see Box 2).

The mechanism proposed here appeals to basic, well-established processes from the associative learning literature to provide a clear and plausible explanation of current findings. The novel step we have taken is to pair an associative account with what we know about the perceived physical similarity elicited by bodily illusions. By appealing to a multidimensional self-representation, in which both bodily and conceptual aspects of the self are bound in a coherent, supramodal construct, we can bridge the gap between the perceptual, bodily representations involved in body ownership, and the evaluative, conceptual representations involved in implicit social attitudes. The resulting mechanism provides us with a coherent account of how changes in body ownership can close this gap in order to affect higher-level social processes.

\section{Concluding Remarks}

Overall, an intriguing and consistent pattern of results has emerged from independent research groups, whereby changes in the experience of ownership over an outgroup body of different race results in significant reductions of the levels of implicit bias against that outgroup . Furthermore, similar changes are elicited in measures of somatosensory remapping [14] that reflect levels of body resonance between people. Taken together, these findings suggest that changes in the perceived similarity between self and others, caused by shared multisensory experiences, might 'bridge the gap' between the basic, perceptual representation of bodies, and the complex social mechanisms underlying much of our everyday social interaction. 
A key challenge for future work is to elucidate the neural mechanisms involved in these changes. The networks involved in implicit racial bias are already known [2], and appear to serve two related yet distinct functions. The first function, likely to be subserved by the amygdala, generates a rapid, automatic affective reaction to other-race stimuli, and the second, subserved by the dorsolateral prefrontal cortex and anterior cingulate cortex, controls and regulates the expression of this affective reaction according to explicitly desired behaviours. Thus, an investigation of the neural mechanisms of our findings will allow us to clearly elucidate exactly which process is being altered; do the changes in body ownership alter the initial affective processing of the racial outgroup, or instead alter the way this affective reaction is detected, controlled and expressed?

A neural investigation of the effects of 'changing race' will also reveal important information regarding its effects on bodily resonance. Initial behavioural evidence has suggested that induced changes in body ownership can increase somatosensory resonance when observing a different-race individual being touched [14]. An important next step would be to investigate if this increased resonance extends to other domains, e.g., the motor domain, where it could have important consequences for key social processes [7]. We propose that changes in perceived interpersonal similarity play a causal role in this mechanism, and it is now timely to elucidate how exactly this interpersonal bodily similarity may modulate activity in the ‘mirror system’.

These recent findings also lead us to new insights into how implicit social biases are formed and maintained. Previously, implicit racial biases have been considered relatively difficult to change [43]. Earlier attempts to alter these racial biases have tended to involve lengthy training programmes and conscious interventions [e.g. 44]. In contrast, the research we have reviewed here has revealed an exciting new mechanism by which implicit social biases can be modulated 'from the body upwards'. We propose a potential cognitive underpinning of these changes, from body ownership to social cognition, which appeals to associative learning and predictive coding to provide us not only with a rich theoretical framework in which the current data can be understood, but also with a set of open questions to test in future research (see Box 3). 


\section{Acknowledgements}

224 MT is supported by the European Research Council (ERC-2010-StG-262853) under the 225 FP7, and the European Platform for Life Sciences, Mind Sciences and Humanities of the 226 Volkswagen Foundation. MS and MVSV are supported by the European Union FP7 Future and 227 Emerging Technologies (FET) Integrated Project VERE (\#257695), and the European Union FP7 228 ICT Integrated Project BEAMING (\#248620). MS is supported by the European Research Council 229 ERC Advanced Grant TRAVERSE (\#227985), the FP7 European Union AAT Program project VR230 HYPERSPACE (\#AAT-285681) and the Spanish Ministerio de Ciencia i Innovación project 231 ResPIVE (\#PSI2011-27930). 


\section{References}

1. Baumeister, R. F., \& Bushman, B. J. (2014). Social Psychology and Human Nature (3rd Edition). Belmont, CA: Wadsworth/ Cengage.

2. Kubota, J. T., Banaji, M. R., Phelps, E. A. (2012). The neuroscience of race. Nature Neuroscience, 15(7), 940-8.

3. Avenanti, A., Sirigu, A., \& Aglioti, S.M. (2010). Racial bias reduces empathic sensorimotor resonance with other-race pain. Current Biology, 20, 1018-1022.

4. Gutsell, J. N., \& Inzlicht, M. (2010). Empathy constrained: Prejudice predicts reduced mental simulation of actions during observation of outgroups. Journal of Experimental Social Psychology, 46(5), 841-845.

5. Serino, A., Giovagnoli, G., \& Làdavas, E. (2009). I Feel what You Feel if You Are Similar to Me. PLoS ONE, 4(3), e4930.

6. Xu, X., Zuo, X., Wang, X., \& Han, S. (2009). Do you feel my pain? Racial group membership modulates empathic neural responses. The Journal of Neuroscience, 29(26), 8525-8529.

7. Gallese, V., Keysers, C., \& Rizzolatti, G. (2004). A unifying view of the basis of social cognition. Trends in Cognitive Sciences, 8(9), 396-403.

8. Desy, M. C., \& Theoret, H. (2007). Modulation of motor cortex excitability by physical similarity with an observed hand action. PLoS ONE, 2(10), e971.

9. Maister, L., Sebanz, N., Knoblich G., \& Tsakiris, M. (2013) Experiencing ownership over a dark-skinned body reduces implicit racial bias. Cognition, 128 (2), 170-178.

10. Peck, T. C., Seinfeld, S., Aglioti, S. M., \& Slater, M. (2013) Putting yourself in the skin of a black avatar reduces implicit racial bias. Consciousness and Cognition. 22(3), 779-87.

11. Banakou, D., Groten, R., Slater, M. (2013). Illusory ownership of a virtual child body causes overestimation of object sizes and implicit attitude changes. Proceedings of the National Academy of Sciences, 110(31), 12846-51.

12. Farmer, H., Maister L., \& Tsakiris, M. (2014). Change my body, change my mind: the effects of illusory ownership of an outgroup hand on implicit attitudes towards that outgroup. Frontiers in Psychology: Cognitive Science, 4, 1016.

13. Farmer, H., Tajadura-Jiménez, A., \& Tsakiris M. (2012). Beyond the colour of my skin: How skin colour affects the sense of body-ownership. Consciousness and Cognition, 21, 1242-56

14. Fini, C., Cardini, F., Tajadura-Jimenez, A., Serino, A. \& Tsakiris, M. (2013) “Embodying an outgroup: the role of racial bias and the effect of multisensory processing in somatosensory remapping” Frontiers in Behavioral Neuroscience, 7,165.

15. Greenwald, A. G., McGhee, D. E., \& Schwartz, J. K. L. (1998). Measuring individual differences in implicit cognition: The Implicit Association Test. Journal of Personality and Social Psychology, 74, 1464-1480. 
16. Tsakiris, M. (2010). My body in the brain: A neurocognitive model of body-ownership. Neuropsychologia, 48, 703-12.

17. Keysers, C., \& Gazzola, V. (2009). Expanding the mirror: Vicarious activity for actions, emotions, and sensations. Current opinion in neurobiology, 19(6), 666-671.

18. Brozzoli, C., Gentile, G., Bergouignan, L., \& Ehrsson, H. H. (2013). A shared representation of the space near oneself and others in the human premotor cortex. Current Biology, 23(18), 1764-1768.

19. Iacoboni, M., Molnar-Szakacs, I., Gallese, V., Buccino, G., Mazziotta, J. C., \& Rizzolatti, G. (2005). Grasping the intentions of others with one's own mirror neuron system. PLoS biology, 3(3), e79.

20. Morrison, I., Lloyd, D., Di Pellegrino, G., \& Roberts, N. (2004). Vicarious responses to pain in anterior cingulate cortex: is empathy a multisensory issue? Cognitive, Affective \& Behavioral Neuroscience, 4(2), 270-278.

21. Heberlein, A. S., \& Atkinson, A. P. (2009). Neuroscientific evidence for simulation and shared substrates in emotion recognition: Beyond faces. Emotion Review, 1(2), 162-177.

22. Botvinick, M., \& Cohen, J. (1998). Rubber hands' feel'touch that eyes see. Nature, 391(6669), 756-756.

23. Petkova, V. I., \& Ehrsson, H. H. (2008). If I were you: perceptual illusion of body swapping. PloS one, 3(12), e3832.

24. Lenggenhager, B., Tadi, T., Metzinger, T., \& Blanke, O. (2007). Video ergo sum: manipulating bodily self-consciousness. Science, 317(5841), 1096-1099.

25. Slater, M., Spanlang, B., Sanchez-Vives, M. V., \& Blanke, O. (2010). First person experience of body transfer in virtual reality. PloS one, 5(5), e10564.

26. Tsakiris, M. (2008). Looking for myself: current multisensory input alters self-face recognition. PloS one, 3(12), e4040.

27. Longo, M. R., Schuur, F., Kammers, M.P., Tsakiris, M., \& Haggard, P. (2009) Self awareness and the body image. Acta Psychologica, 132(2), 166-172.

28. Bufalari, I. et al. (2014) Enfacing others but only if they are nice to you. Front. Behav. Neurosci. 8, 102

29. Kilteni, K., Bergstrom, I., and Slater, M. (2013). Drumming in immersive virtual reality: the body shapes the way we play. IEEE transactions on visualization and computer graphics 19, 597-605.

30. van der Hoort, B., Guterstam, A., \& Ehrsson, H. H. (2011). Being Barbie: the size of one's own body determines the perceived size of the world. PLoS One, 6(5), e20195.

31. Preston, C., \& Ehrsson, H. H. (2014). Illusory Changes in Body Size Modulate Body Satisfaction in a Way That Is Related to Non-Clinical Eating Disorder Psychopathology. PloS one, 9(1), e85773. 
32. Normand, J.M., Giannopoulos, E., Spanlang, B., and Slater, M. (2011). Multisensory Stimulation Can Induce an Illusion of Larger Belly Size in Immersive Virtual Reality. PLoS ONE 6, e16128.

33. Paladino, M. P., Mazzurega, M., Pavani, F., \& Schubert, T. W. (2010). Synchronous multisensory stimulation blurs self-other boundaries. Psychological Science, 21(9), 12021207.

34. Maister, L., Tsiakkas, E., \& Tsakiris, M. (2013). I feel your fear: shared touch between faces facilitates recognition of fearful facial expressions. Emotion, 13(1), 7-13.

35. Sforza, A., Bufalari, I., Haggard, P., \& Aglioti, S. M. (2010). My face in yours: Visuotactile facial stimulation influences sense of identity. Social neuroscience, 5(2), 148-162.

36. Tajadura-Jiménez, A., Grehl, S., \& Tsakiris, M. (2012). The other in me: interpersonal multisensory stimulation changes the mental representation of the self. PloS one, 7(7), e40682.

37. Tao, R., Zhang, S., Li, Q., \& Geng, H. (2012). Modulation of self-esteem in self-and otherevaluations primed by subliminal and supraliminal faces. PloS one, 7(10), e47103.

38. Ma, Y., \& Han, S. (2010). Why we respond faster to the self than to others? An implicit positive association theory of self-advantage during implicit face recognition. Journal of Experimental Psychology: Human Perception and Performance, 36(3), 619.

39. Field, A. P., \& Davey, G. C. (1999). Reevaluating evaluative conditioning: A nonassociative explanation of conditioning effects in the visual evaluative conditioning paradigm. Journal of Experimental Psychology: Animal Behavior Processes, 25(2), 211.

40. De Houwer, J., Thomas, S., \& Baeyens, F. (2001). Association learning of likes and dislikes: A review of 25 years of research on human evaluative conditioning. Psychological bulletin, 127(6), 853.

41. Gawronski, B., \& Quinn, K. A. (2013). Guilty by mere similarity: Assimilative effects of facial resemblance on automatic evaluation. Journal of Experimental Social Psychology, 49(1), $120-125$.

42. Verosky, S. C., \& Todorov, A. (2010). Generalization of affective learning about faces to perceptually similar faces. Psychological Science, 21(6), 779-785.

43. Joy-Gaba, J. A., \& Nosek, B. A. (2010). The surprisingly limited malleability of implicit racial evaluations. Social Psychology, 41(3), 137-146.

44. Devine, P. G., Forscher, P. S., Austin, A. J., \& Cox, W. T. (2012). Long-term reduction in implicit race bias: A prejudice habit-breaking intervention. Journal of experimental social psychology, 48(6), 1267-1278.

45. Gallagher, S. (2000). Philosophical conceptions of the self: implications for cognitive science. Trends in Cognitive Sciences, 4, 14-21.

46. Baumeister RF. The self. In: Gilbert DT, Fiske ST, Lindzey G, editors. The Handbook of Social Psychology. 4th Boston, MA: McGraw-Hill; 1998. pp. 680-740. 
47. Tsakiris, M., \& Haggard, P. (2005). The rubber hand illusion revisited: visuotactile integration and self-attribution. Journal of Experimental Psychology: Human Perception and Performance, 31(1), 80.

48. Ehrsson HH, Weich K, Weiskopf N, Dolan RJ and Passingham RE. Threatening a rubber hand that you feel is yours elicits a cortical anxiety response. Proc. Natl. Acad. Sci. USA (2007), 104, 9828-9833.

49. Slater, M., Perez-Marcos, D., Ehrsson, H. H., \& Sanchez-Vives, M. V. (2008). Towards a digital body: the virtual arm illusion. Frontiers in human neuroscience, 2,6.

50. Sanchez-Vives, M. V., Spanlang, B., Frisoli, A., Bergamasco, M., \& Slater, M. (2010). Virtual hand illusion induced by visuomotor correlations. PloS one, 5(4), e10381.

51. Kilteni, K., Normand, J. M., Sanchez-Vives, M. V., \& Slater, M. (2012). Extending body space in immersive virtual reality: a very long arm illusion. PloS one, 7(7), e40867.

52. Perez-Marcos, D., Sanchez-Vives, M. V., \& Slater, M. (2012). Is my hand connected to my body? The impact of body continuity and arm alignment on the virtual hand illusion. Cognitive Neurodynamics, 6(4), 295-305.

53. Tajadura-Jiménez, A., Longo, M. R., Coleman, R., \& Tsakiris, M. (2012). The person in the mirror: using the enfacement illusion to investigate the experiential structure of selfidentification. Consciousness and cognition, 21(4), 1725-1738.

54. Slater, M., and Sanchez-Vives, M.V. (2014). Transcending the Self in Immersive Virtual Reality. IEEE Computer 47, 24-30.

55. Apps, M. A., \& Tsakiris, M. (2014). The free-energy self: a predictive coding account of self-recognition. Neuroscience \& Biobehavioral Reviews, 41, 85-97.

56. Seth, A.K. (2013). Interoceptive inference, emotion, and the embodied self. Trends in Cognitive Sciences, 17(11), 656-663.

57. Moutoussis, M., Fearon, P., El-Deredy, W., Dolan, R. J., \& Friston, K. J. (2014). Bayesian inferences about the self (and others): a review. Consciousness and cognition, 25, 67-76. 


\section{Glossary}

Bodily resonance: The process by which the perception of bodily states in others can activate similar bodily states in the self $[5,17,18]$. This process is thought to be central to a number of fundamental social processes including empathy, action understanding and emotion recognition. This can be measured at the neural level, for example by recording activity in the premotor cortex when observing other-performed actions [17], or behaviourally, for example by measuring the increase in a participant's tactile sensitivity caused by observing another being touched [5].

Body ownership: Body ownership refers to the special perceptual status of one’s own body, which makes bodily sensations seem unique to oneself, that is, the feeling that “my body" belongs to me, and is ever present in my mental life $[16,45]$.

Implicit association task (IAT): The IAT is a computerised task which involves a rapid categorisation of verbal stimuli, pictorial stimuli, or both. Analysis of the patterns of response times and errors provides a metric of implicit associations between categories. Commonly, the associations measured are between a social category, e.g., a specific racial group, and positive versus negative associations, to provide a measure of bias in implicit evaluative attitudes. Implicit biases measured using this method have been found to be internally consistent, reliable and predictive of explicit behaviours [15].

Self-concept: A multidimensional construct, comprising a collection of knowledge structures regarding one's attitudes, dispositions, skills and abilities, which are temporally stable and transsituational [46]. 


\section{Box 1: Manipulations of Body Ownership}

\section{Rubber Hand Illusion (RHI)}

Watching a rubber hand being stroked synchronously with one’s own unseen hand causes the rubber hand to be attributed to one's own body, to "feel like it’s my hand" [22]. This synchronous stimulation not only elicits a subjective experience of ownership over the hand, but also causes the perceived location of one's own hand to drift towards the rubber hand [47] and a stress-evoked skin conductance response to be elicited when the rubber hand is threatened [48]. The illusion of ownership over the rubber hand does not occur when the rubber hand is stroked asynchronously with respect to the subject's own hand, and thus experiments investigating body ownership commonly use asynchronous stimulation as a control condition. An illusion of the same intensity can be also developed over a virtual hand by either synchronous visuotactile [49] or visuomotor [50] correlations. This illusion persists through radical transformations such as extensive elongation of the arm [51] or change in the virtual hand position [52] with respect to the real one.

\section{Enfacement Illusion}

The enfacement illusion is a facial analogue of the rubber hand illusion. Participants watch a video showing the face of an unfamiliar other being stroked with a cotton bud on the cheek, while the participant receives identical stroking on their own, congruent cheek in synchrony with the touch they see. As in the RHI, synchronous, but not asynchronous, visuotactile stimulation elicits illusory feelings of ownership over the other’s face [53]. Enfacement also influences social cognition $[33,34]$ and produces a measurable bias in self-face recognition, whereby participants perceive the other's face as looking more like their own [26,35,36].

\section{Full-body illusions}

Illusory ownership over a physical manikin body that substituted the participant's real body was demonstrated in [23]. Live video, from cameras attached to the manikin, was streamed to head- 
417 mounted displays on the participants, so that when looking down they would see the manikin body

418 visually substituting their own. Synchronous tapping on the manikin body and the real body led to

419 illusory body ownership, in a similar way to the more traditional rubber hand and enfacement

420 illusions. More advanced systems have now been developed, using Immersive Virtual Reality (IVR)

421 [25]. Participants wear a head-tracked stereo head-mounted display which provides computer

422 generated images immersing the participant in a virtual world. The participant's own body is

423 substituted by a virtual body, viewed from a first-person perspective, with a motion capture system

424 so that their virtual body moves with their real body movements. This set up results in sensorimotor 425 correlations (visual, proprioceptive, tactile and motor) that elicit illusions of ownership and agency 426 over the virtual body $[10,11,54]$. 
427 Figure 1. Inducing ownership over a body of another race

428 A. The Rubber Hand Illusion: Light-skinned Caucasian participants observe a dark-skinned rubber 429 hand being stimulated in synchrony with their own unseen hand. This elicits a shift of body 430 ownership to incorporate the other-race limb [adapted from 9].

431 B. The Enfacement Illusion: Participants viewed the face of a racial outgroup member being 432 stimulated in synchrony with their own to induce a sense of ownership over the observed face [see $43314]$.

434 C. Immersive Virtual Reality: (i) A participant wears a wide field-of-view stereo head-tracked head435 mounted display and a motion capture suit for real time body tracking. (ii) This is the participant's 436 view of the situation, whereby she can see her virtual body both directly and reflected in the mirror, 437 in stereo as shown. The body she sees could be dark-skinned, light-skinned or purple; in this case, 438 the virtual body is dark-skinned whereas she is light-skinned [adapted from 10]. 
440 Figure 2: Changes in implicit racial attitudes after incorporating an other-race body in an 441 Immersive Virtual Reality setup

442 Light-skinned Caucasian participants took part in a between-groups experiment where they 443 occupied a White (A) or Black (B) body in a virtual environment. They could see their body from a 444 first-person perspective when they looked down, as well as in a virtual mirror (see Figure 1, Panel 445 C(ii)). Two control groups were also included - in these conditions, participants either had no 446 virtual body (C), or the body was of an unnatural purple colour (D) to control for general 447 dissimilarity to their own skin. Participants’ implicit racial biases were measured before and after 448 embodiment. Participants who embodied a Black avatar showed a decrease in their implicit biases 449 against Black individuals, which was significantly greater than for those who embodied a White 450 avatar. Adapted from [10]. 


\section{Box 2: Predictive coding models of the Self}

How we recognise ourselves, and what governs our sense of ownership over our bodies, is still under much debate in the psychological and neuroscientific literature. However, a recent interest in Predictive Coding as a unifying theory of brain function has provided a plausible framework for understanding the cognitive basis of self-recognition [55]. On this account, one’s body is processed in a probabilistic manner as the most likely to be "me", given prior knowledge about our bodies and incoming sensory information. Such probabilistic representation arises through the integration of information from hierarchically organised unimodal systems in higherlevel multimodal areas. In the case of bodily illusions, viewing touch on a different body evokes a sensation of touch on one's own body, and this generates bottom-up error signals from unimodal sensory systems. Perceptual learning processes will update the body representation to first induce a sense of ownership over the new body and next to incorporate perceptual features of the other's body, in order to minimise this error and maintain a continual sense of 'mineness'. Therefore, this account can explain how synchronous multisensory stimulation, such as that provided during the Rubber Hand and other bodily illusions, can not only elicit fundamental shifts in body ownership, but can also elicit a subsequent increase in perceived similarity between the bodies of self and other. Importantly, the self is not represented solely at a basic, perceptual level. The self is a multimodal, hierarchical construct containing both low-level, bodily representations as well as higher level attitudes and beliefs. On a predictive coding account, these different levels of representation continuously interact $[55,56]$, as prediction errors, when left unexplained at one level ,need to be processed and eliminated at a higher level of the hierarchy. An explanatory strength of the predictive coding approach is that it can be applied to the whole information processing hierarchy [e.g. 57], as it argues for complimentary hierarchical top-down and bottom-up processes. A change in low-level, perceptual representations of one's own body in relation to the body of an outgroup member creates errors further up in the processing hierarchy, as this new information now conflicts with more abstract, higher-order representations of oneself and the outgroup. These errors 
478 must then be minimised in a similar way, by updating attitudes and beliefs held about oneself and 479 the outgroup. In this way, the consistency within the multimodal self-representation is maintained. 


\section{$480 \quad$ Box 3: Outstanding Questions}

481 - What is the time course of these effects? Are they persistent over time?

482

- What are the underlying neural mechanisms?

483 - Do these changes in implicit associations have behavioural consequences in daily life?

484 - Can similar effects be found with social groups that are not defined by perceptual differences, such as political or religious groups, merely by informing the participant of the embodied individual's group membership? 
A.

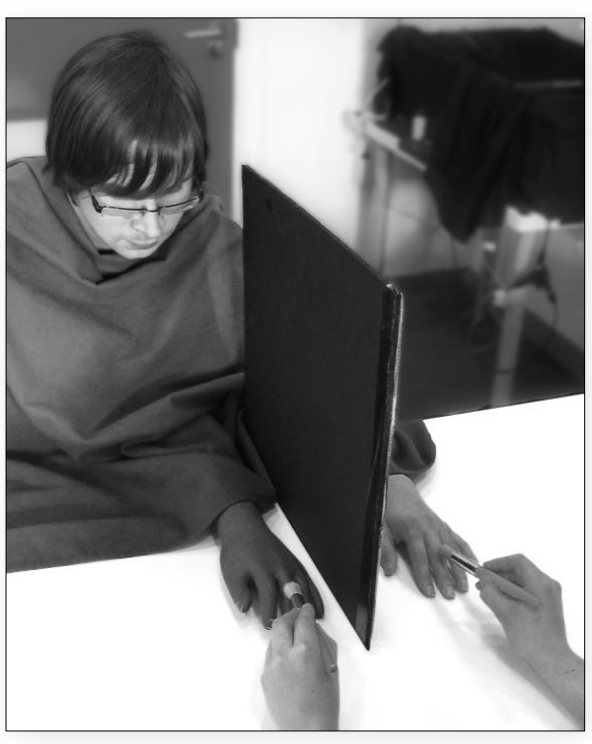

c.

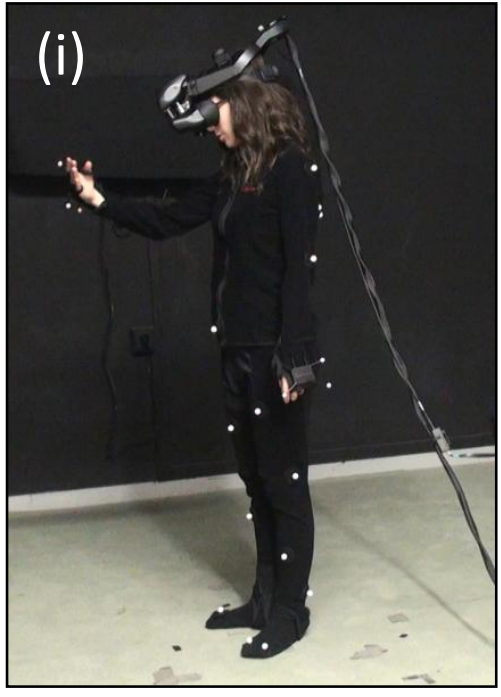

B.

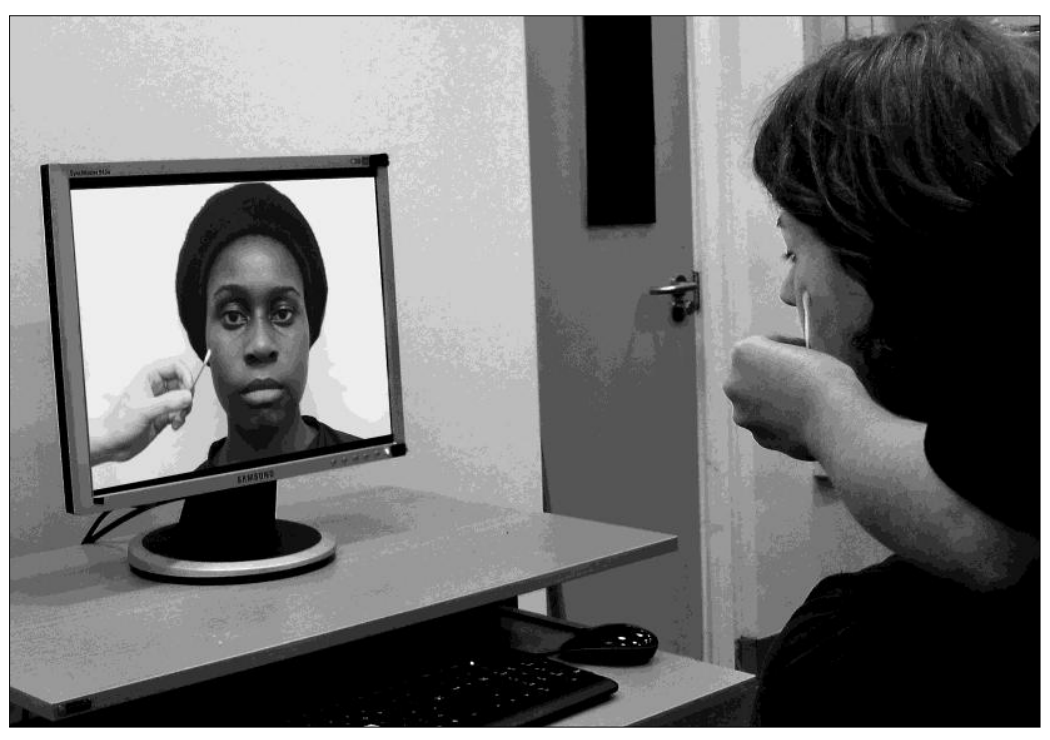

(ii)

Left eye

Right eye 


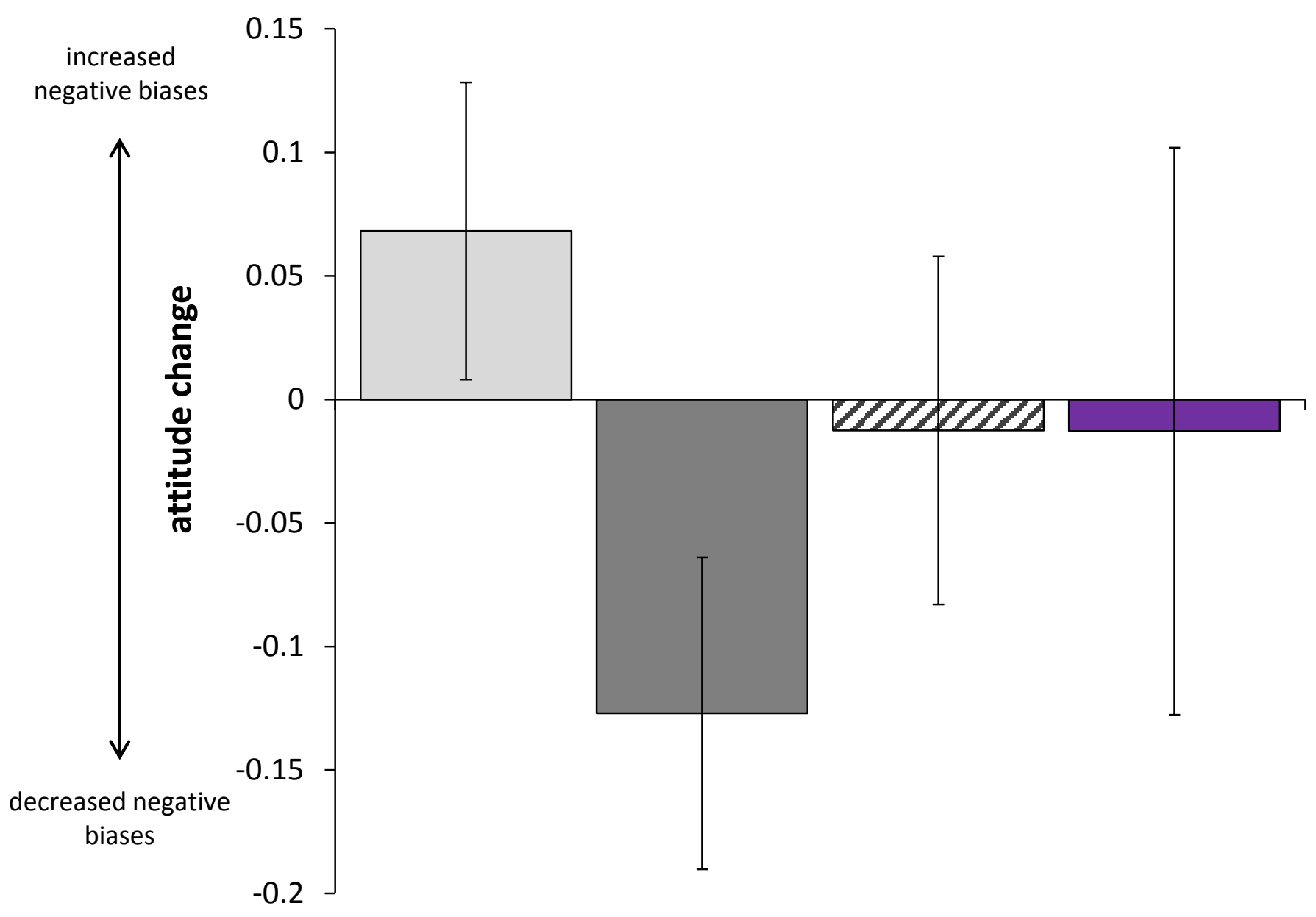

Avatar Embodiment Condition:

$\square$ White

$\square$ Black

口 No Embodiment

- Purple 
From:

Sent:

To:

Subject: em.tics.0.3ee3d3.c7d12363@editorialmanager.com on behalf of Trends in Cognitive Sciences Editorial Office <em@editorialmanager.com>

Sunday, November 02, 2014 7:05 PM

Tsakiris, Manos

Trends in Cognitive Sciences - Manuscript accepted TICS-D-14-00309R1

\section{CC: rschwarzlose@cell.com}

02 Nov 2014

Ref.:

Ms. No. TICS-D-14-00309R1

Changing Bodies Changes Minds: Owning another body affects social cognition Trends in Cognitive Sciences

Dear Dr Tsakiris,

Thank you for submitting your revised manuscript and for all your work on the revision. I have now gone through the revised text, figures, and your responses to reviewer and editorial comments -- the manuscript is stronger and clearer as a result of the revisions and I have no further requests for changes to make. We can therefore move forward with acceptance and publication of your article in Trends in Cognitive Sciences.

Your article will be published online, ahead of print; in advance of this a proof of your article will be made available to you for correcting within the next month. As it is important that either you or a representative will be available during this time to check the proofs, please let me know if you will be away during this time.

** Note, to ensure that your article is accessible to a broad audience, please be aware that your text will be further edited for grammar and clarity following its acceptance for publication. Although the person performing these edits is a professional copy editor, it is vital that you read the proofs carefully to make sure that any changes made have not unintentionally altered the scientific meaning of your text.

As a thank you for writing for us, we offer a free subscription to a Trends journal for one year. To activate this, please reply to this email. Simply select one title from the list given below and email back your full name, full address details and preferred subscription start date for the subscription to be authorized.

Trends in: Biochemical Sciences; Biotechnology; Cell Biology; Cognitive Sciences; Ecology and Evolution; Endocrinology and Metabolism; Genetics; Immunology; Microbiology; Molecular Medicine; Neurosciences; Parasitology; Plant Science; Pharmacological Sciences

Please bear in mind that you will be provided with an electronic PDF version of your article once it has been published: this will come direct to you from ScienceDirect when the issue you have written for is compiled online.

If you have any feedback for us regarding your interaction with the editorial office, please let me know.

Thank you again for a fascinating contribution to Trends in Cognitive Sciences.

With all best wishes,

Becca

Rebecca Schwarzlose, PhD 
Editor

Trends in Cognitive Sciences

No virus found in this message.

Checked by AVG - www.avg.com

Version: 2014.0.4765 / Virus Database: 4189/8497 - Release Date: 11/02/14 